\title{
Performance Analysis of Data Traffic Offload Scheme on Long Term Evolution (LTE) and IEEE 802.11 AH
}

\author{
Made Adi Paramartha Putra ${ }^{* 1}$, Doan Perdana ${ }^{2}$, Ridha Muldina Negara ${ }^{3}$ \\ Telecommunication Engineering, School of Electrical Engineering, Telkom University \\ Bandung, Indonesia \\ ${ }^{*}$ Corresponding author, e-mail: mdparamartha@gmail.com ${ }^{1}$, doanperdana@telkomuniversity.ac.id ${ }^{2}$, \\ ridhanegara@telkomuniversity.ac.id ${ }^{3}$
}

\begin{abstract}
One of new mobile technology is being developed by 3GPP is Long Term Evolution (LTE). LTE usually used by user because provide high data rate. Many traffic sending over LTE, makes several users didn't get good Quality of Service (QoS). Traffic diversion is needed to increasing QoS value. It can be done with offloading data method from LTE to Wi-Fi network. This paper using 802.11ah standard to evaluate Wi-Fi network. IEEE 802.11ah have 1000 meters coverage area and efficiency energy mechanism, which is proposed for M2M in 5G techonology. Some research has proven that traffic diversion with offloading can increasing network performance. The contribution of this paper is to evaluate the impact of traffic offload between LTE and IEEE 802.11ah standard. This paper propose two scenarios using increment number of user and increment mobility speed of user to evaluate throughput and delay value before and after the offload process. The simulation will simulate using Network Simulator-3. We can conclude that network performance after offloading is better for every scenario. For increment number of user scenario, throughput value increasing $29.08 \%$, and delay decreasing $8.12 \%$. Scenario with increment mobility speed of user obtain throughput value increasing $37,57 \%$, and delay value decreasing $27.228 \%$.
\end{abstract}

Keywords: LTE, IEEE 802.11ah, offloading data traffic, throughput, delay

Copyright $\odot 2017$ Universitas Ahmad Dahlan. All rights reserved.

\section{Introduction}

The main issue of wireless technology development is flexibility. One of new standar in wireless is IEEE 802.11ah, which is provide low energy consumption, handling many user, and higher coverage area up to 1000 meters. In other side, our cellular phone using 3GPP wireless standard called 4G Long Term Evolution. LTE technology support high data rate system and high flexibility [1-2]. Based on Cisco data [3] indicate that celluler traffic is increasing $81 \%$ in 2013. 45\% cellular traffic from LTE network diverse into Wi-Fi network and higher 14.5 times than other network standard. Cisco data shows the development of LTE network growth very fast. Traffic diversion from LTE to Wi-Fi is one of technique to increasing the network performance and availability [4]. Traffic diversion from LTE to Wi-Fi network usually called as offloading. Beside, the study of IEEE 802.11ah on offloading between IEEE 802.11ah and LTE infrequently done. This paper propose to evaluate the performance analysis of offloading between IEEE 802.11ah and Long Term Evolution. Moreover, based on study [5] increasing cellular network coverage using Wi-Fi network is more cheap than upgrading existing network.

Masek et al. [6] proposed offload data traffic from LTE network into unlicensed 802.11 links which is simulated using Network Simulator 3 . In this work, they introduce two condition as decision maker in offloading process. Signal to Noise Ratio (SNR) is used in first condition. Meanwhile in second condition they using throughput value. In the result, both condition gives better network performance especially thorughput value. Lee et al. [7] studied about economic benefits from Wi-Fi offloading, which is delay value. Due to many overloads in cellular network, Wi-Fi offloading can reduce mobile data explosion. In their paper detailed that Wi-Fi offloading give economic benefit for users and provider, which increase $131 \%$ in provider side and also increase $246 \%$ in the users side.

Analytical models on the Wi-Fi offloading efficiency was developed by Suh et al. [8]. WiFi offloading was the promising technique to solve mobile data explosion. Furthermore, there are two different types of offloading techniques that described. Opportunistic Wi-Fi offloading 
and delayed $\mathrm{Wi}-\mathrm{Fi}$ offloading. Using the analytical model and simulation, they obtain that Wi-Fi offloading can improve network efficiency. Based on the several studies above, Wi-Fi offloading is one solution to increasing the network performance and availability due to many LTE users didn't acquires good Quality of Services (QoS). The contribution of this paper is to increasing network performance using traffic offload between LTE and IEEE 802.11ah standard.

IEEE 802.11ah is new standard for M2M and WSN which proposed in 5G technologies [9]. Moreover IEEE 802.11ah supports large coverage area up to 1000 meters and using Restricted Access Window (RAW) mechanism to minimize the energy consumption [10]. To obtain better network performance, IEEE 802.11ah is used in our work to evaluate the network performance before and after offload process. We also using Network Simulator-3 to simulate network condition and check QoS value, especially throughput and end-to-end delay value.

This paper organized as follows: Section 2 gives an overview about the algorithm and the scenario. Result of performance evaluation will discribed in section 3 as well with analysis. And section 4 provides the conclusions and suggestion for future work.

\section{Research Method}

This paper focused to evalute network performance before and after offload process. This research use Network Simulator-3 dev version as simulation tool which is used with LTE module. IEEE 802.11ah module that we use has been developed by [11]. In this paper, traffic generator is using Constant Bit Rate (CBR) and User Datagram Protocol (UDP) module.

The simulation system is built by two scenarios which are offloading with increment number of user and offloading with increment of user mobility speed. In each scenario we will evaluate end-to-end delay and throughput value to obtain how offload process affected network performance.

\subsection{Offloading with Increment Number of User}

First scenario is using a condition in which total number of user increasing. The user increment start from 10 user, 20 user, 30 user, 40 user and 50 user. While the total number of user increasing, the mobility speed model of each increment in constant position. The mobility speed of user is $0 \mathrm{~km} /$ hours.

Table 1. Offloading with Increment Number of User

\begin{tabular}{ccc}
\hline Scenario & Mobility Speed $(\mathrm{Km} / \mathrm{h})$ & Number of User \\
\hline & & 10 \\
Increment & 0 & 20 \\
Number of User & 0 & 30 \\
& & 40 \\
& & 50 \\
\hline
\end{tabular}

\subsection{Offloading With Increment of User Mobility Speed}

Another scenario condition is increment of user mobility speed. I this scenario we increasing the mobility speed from each user. Mobility speed increment start from $1 \mathrm{~km} /$ hours, $2 \mathrm{~km} /$ hours, $3 \mathrm{~km} /$ hours, $4 \mathrm{~km} /$ hours and $5 \mathrm{~km} /$ hours. While the mobility speed of user is increasing, total number of user is constant in 10 user for every mobility speed.

Table 2. Offloading with Increment of User Mobility Speed

\begin{tabular}{ccc}
\hline Scenario & Mobility Speed $(\mathrm{Km} / \mathrm{h})$ & Number of User \\
\hline & 1 & \\
Increment of User & 2 & 10 \\
Mobility Speed & 3 & \\
& 4 & \\
\hline
\end{tabular}




\subsection{Flowchart System}

Based on previous paper [6], which is checking network performance only before the offload process based on SNR and throughput value. In this paper we using throughput value as decision maker, we also evaluate the network performance after the offload process.

Figure 1 descibe the offload process and decision. User will choose an interface that provide higher throughput value. Simulation time before offload process same take 1 second of simulation time, and after offload process take another 1 second. Each scenario done by doing 60 times simulation. There are some related parameter that we use in this simulation.

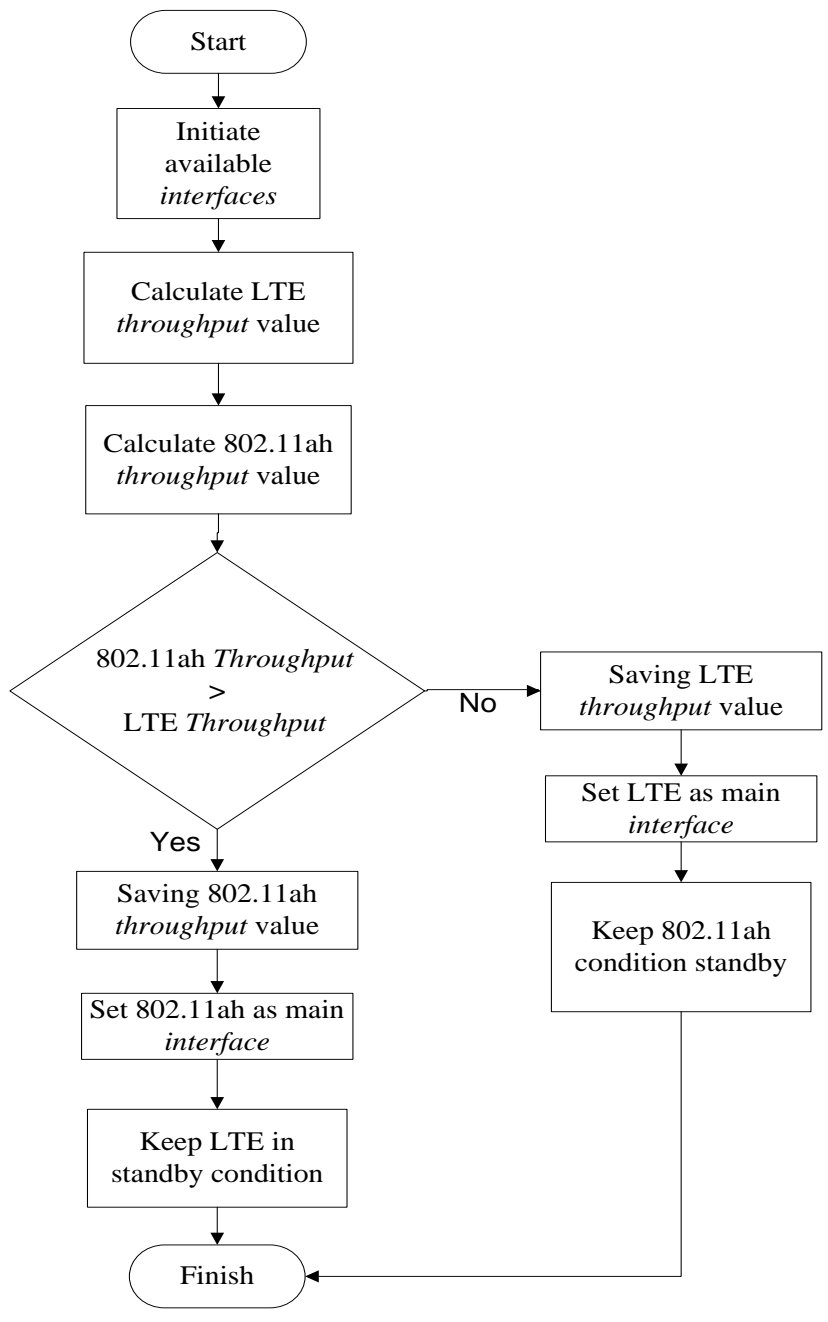

Figure 1. System for Wi-Fi Offloading

Table 3. Simulation Parameters

\begin{tabular}{ccccc}
\hline No & Parameters & Units & LTE & IEEE 802.11ah \\
\hline 1 & Operating Frequency & MHz & 1800 & \multicolumn{2}{c}{900} \\
2 & Transport Layer & & & UDP \\
3 & Packet Size & Bytes & \multicolumn{2}{c}{1024} \\
4 & Data Rate Traffic & Mbps & 1 & 1 \\
5 & Bandwidth & MHz & 1,4 & 5 \\
6 & RAW Group & Group & - & 200 \\
7 & Distance eNB-AP & Meter's & 10 & \\
8 & User Distance & Meter's & \multicolumn{2}{c}{ 10, 20, 30, 40, 50 } \\
9 & Number of User & User & \multicolumn{2}{c}{ 1, 2, 3, 4, 5 } \\
10 & Mobility Speed & km/hours & Random Direction 2D Mobility Model \\
11 & Mobility Model & - & Randon
\end{tabular}


These parameter values are sets in NS-3 dev as simulation tool. Several parameters settings above is used for LTE and IEEE 802.11ah network. Furthermore these parameter also used in both scenario, offloading with increment number of user and offoading with increment mobility speed of user. Both simulation scenario is conducted to achieve the purpose of the reserach, which is to evaluate network performance on offloading between Long Term Evolution and IEEE 802.11ah standard.

\section{Results and Analysis}

We use several performance parameters such as network throughput and end-to-end delay to achieve the goal of this research. The result will diveded into two parts, before and after offload process. Before offload is a condition without offloading process, and after offload is a condition with offload process between IEEE 802.11ah and Long Term Evolution.

\subsection{Throughput Measurement}

Throughput is the average data rate successfully received by the recipient [12]. These parameter shows how much actual data can be send over network. Throughput is measured in units of data size per time. The units used are Kilobits per second. Throghput value can be calculated using this formula

$$
\text { Throughput }=\frac{\text { Ereceived packets size }}{\text { time duration }}
$$

Figure 2 shows that throughput value always increasing after offload process. Based on scenario with increment number of user, when the user condition increasing affected throughput value decreasing. Decrement of throughput value due to many user accessing the network at the same time. Before offload process we obtain average throughput value $205,086714 \mathrm{Kbps}$. And after the offload process we obtain the average throughput value of user is 264,74485 Kbps. Average throughput value before and after the offload process increasing 29,08 \%. But after the offload process, throughput value decreasing and its due many number of user accessing the network too.

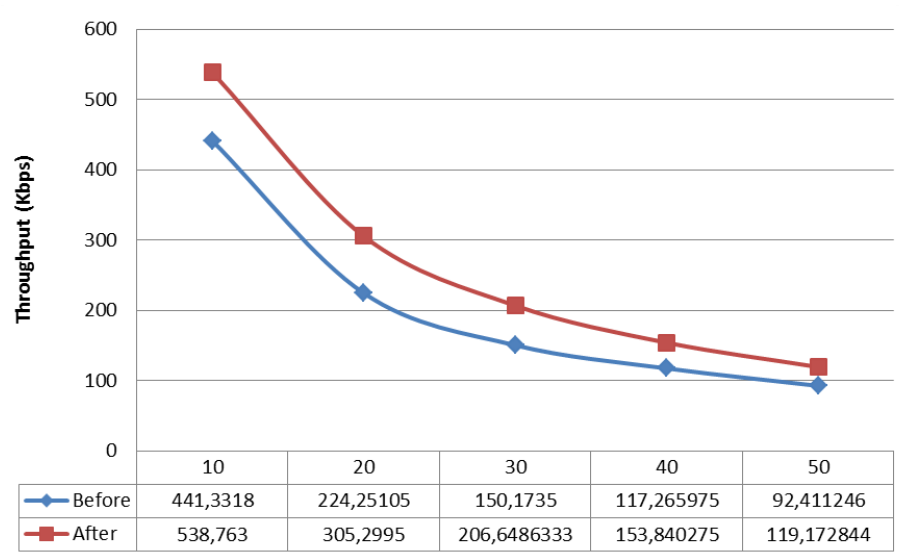

Figure 2. Throughput value with increment number of user

Figure 3 shows throughput value before and after offload process in condition with increment of user mobility speed, which is after the offload process we obtain better throughput value. Before offload process we obtain average thorughput 456,67886 Kbps. And after the offload process we obtain the average throughput is increasing to $628,26704 \mathrm{Kbps}$. So the average throughput after the offload process is increasing $37,57 \%$. Based on figure 3 , in condition with increment of user mobility speed we can see that the mobility speed from 1 
$\mathrm{km} /$ hours to $5 \mathrm{~km} /$ hours didnt affect to much to throughput value. But if we considering from the average value, offload process gives higher throughput value.

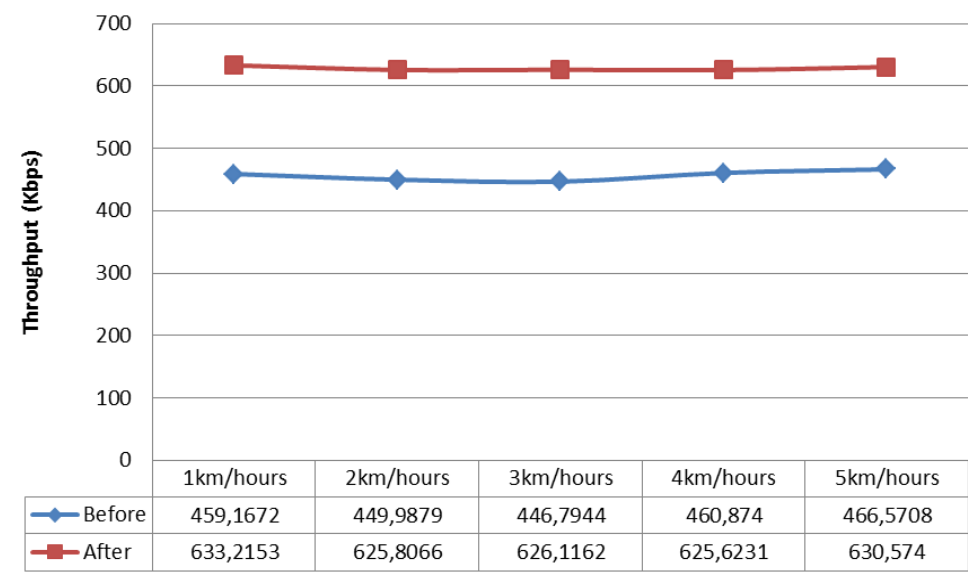

Figure 3. Throughput value with increment of user mobility speed

\subsection{End-to-end Delay Measurement}

End-to-end delay is the time from packet send from sender to received in final destination. End-to-end delay can be affected by distance, transmission model and even propagation or queuing. End-to-end delay mathematically formula can be written as follows.

$$
\text { End }- \text { to }- \text { end delay }=\frac{\text { received packets time }- \text { sent packets time }}{\sum \text { received packets }}
$$

Figure 4 shows that the value of end-to-end delay on first scenario. We obtain average end-to-end delay value with increment number of user before offload process 353,191935 ms. After the offload process we found the average end-to-end delay value is decreasing to 324 , $500027 \mathrm{~ms}$. So the average end-to-end delay after the offload process is dereasing $8,12 \%$. In Figure 4 also shows that when the condition of user is increase, end-to-end delay value also increasing. This problem also caused by to many user accessing the network, so each user should be waiting before get serviced.

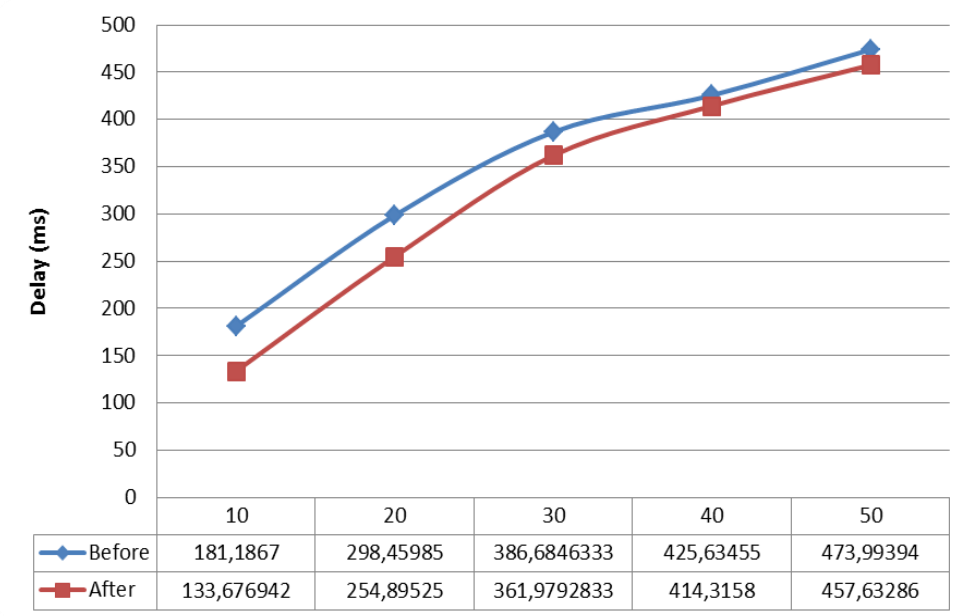

Figure 4. End-to-end delay value with increment number of user 
Figure 5 shows end-to-end delay value for condition with increment of user mobility speed. Its shows that the average end-to-end delay value before offload process is 162,2549 ms. And after the offlad process done, we obtain the average end-to-end delay value decreasing to $117,97869 \mathrm{~ms}$. The decrement of average end-to-end delay value is about 27,228 $\%$. Figure 5 also shows that the mobility speed of user didnt affect to much to end-to-end delay value just like throughput value. But frequently when the mobility speed is increasing, throughput value is decreasing.

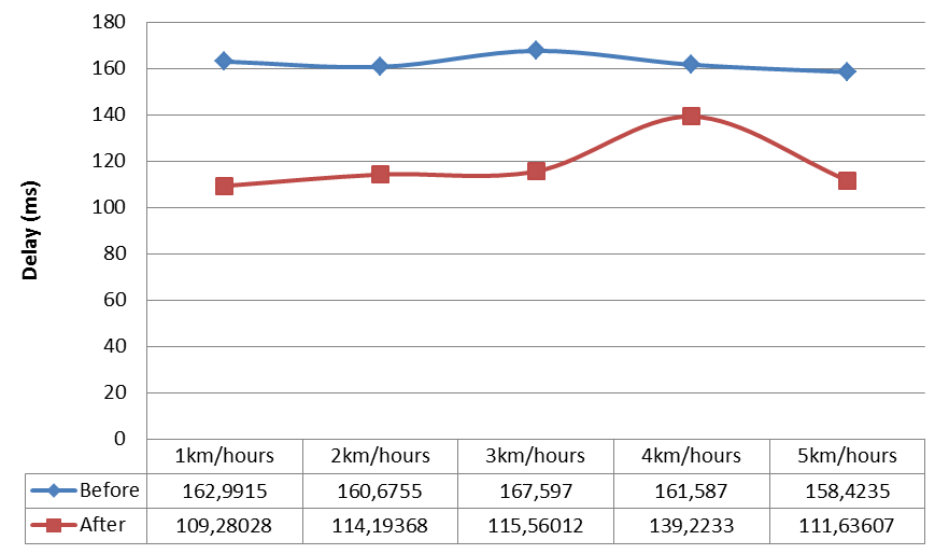

Figure 5. End-to-end delay value with increment of user mobility speed

\section{Conclusion and Future Work}

This research concludes that the offload process between LTE and IEEE 802.11ah network is provide better network performance, especially on throughput and end-to-end delay value. Throughput value always increasing for increment number of user and increment mobility speed of user, as well as end-to-end delay value is decreasing.

After the offload process we obtain throughput value is increasing $29,08 \%$ for increment number of user, and $37,57 \%$ for increment mobility speed of user. This enchancement is very good to improve the network performance. Moreover end-to-end delay value after offload process also decreasing. For first scenario with increment number of user we obtain end-to-end delay value decreasing $8,12 \%$ after the offload process. Also in a condition with increment of mobility speed of user, end-to-end delay value decreasing 27,228 $\%$. Both throughput and end-to-end delay value of network shows an improvement after the offload process done.

For next research, we suggest that the measurement of offloading done with higher number of users, higher mobility speed and even energy consumption spent by user. Considering IEEE 802.11ah standard provide by RAW mechanism to make efficient use of energy.

\section{References}

[1] Iskandar, Hidayat D. SC-FDMA LTE Performance through High Altitude Platforms Communication (HAPS) Channel. Journal TELKOMNIKA. 2016.

[2] Hassan RS, Rahman TA, Abdulrahman AY. LTE Coverage Network Planning and Comparison with Different Propagation Models. Journal TELKOMNIKA. 2014.

[3] Cisco. Cisco visual networking index: Global mobile data traffic forecast update, 2013-2018. 2014.

[4] S Dimatteo, P Hui, B Han, V Li. Cellular traffic offloading through WiFi networks. Proceedings of the IEEE International Conference on Mobile Ad-hoc and Sensor Systems (MASS), Spain. 2011.

[5] A Aijaz, H Aghvami, M Amani. A survey on mobile data offloading: technical and business perspectives. Wireless Communications, IEEE. 2013; 20(2): 104-112.

[6] Masek P, Zeman K, Hosek J, Tinka Z, Makhlouf N, Muthanna A, Herencsar N, Novotny V. User Peformance Gains by Data Offloading of LTE Mobilte Traffic onto Unlicensed IEEE 802.11 Links. IEEE. 2015. 
[7] J Lee, Y Yi, S Chong, Y Jin. Economics of WiFi Offloading: Trading Delay for Cellular Capacity. IEEE Transactions on Wireless Communications. 2014; 13(3): 1540-1554.

[8] Dongeun S, Haneul K, Sangheon P. Efficiency Analysis of WiFi Offloading Techniques. IEEE Transactionson Vehicular Technology. 2015.

[9] Hinger D, Kalbande D. Investigation of Throughput Gains by Mobile data offloading from LTE to WiFi. In Annual IEEE India Conference (INDICON), 2014.

[10] Denatama MI, Perdana D, Negara RM. Analisis Perbandingan Kinerja Protokol Routing DSDV dan OLSR Untuk Perubahan Kecepatan Mobilitas pada Standar IEEE 802.11ah. Jurnal Infotel. 2016; 8(2): 100-106.

[11] Le T, Steven L, Jeroen F. An IEEE 802.11ah simulation module for NS-3. University of Antwerp iMinds. 2016.

[12] Perdana D, Sari RF. Performance Evaluation of Multi-channel Operation IEEE 1609.4 Based on Multi-hop Dissemination. IJCSNS International Journal of Computer Science and Network Security. 2013; 13(3): 42. 